\title{
Oncology Clinicians' Defenses and Adherence to Communication Skills Training with Simulated Patients: an Exploratory Study
}

\author{
Mathieu Bernard • Yves de Roten • \\ Jean-Nicolas Despland • Friedrich Stiefel
}

Published online: 26 April 2012

(C) Springer Science+Business Media, LLC 2012

\begin{abstract}
The aim of this exploratory study was to assess the impact of clinicians' defense mechanisms - defined as self-protective psychological mechanisms triggered by the affective load of the encounter with the patient - on adherence to a communication skills training (CST). The population consisted of oncology clinicians $(N=31)$ who participated in a CST. An interview with simulated cancer patients was recorded prior and 6 months after CST. Defenses were measured before and after CST and correlated with a prototype of an ideally conducted interview based on the criteria of CST-teachers. Clinicians who used more adaptive defense mechanisms showed better adherence to communication skills after CST than clinicians with less adaptive defenses $(F(1,29)=5.26, p=0.03, d=0.42)$. Improvement in communication skills after CST seems to depend on the initial levels of defenses of the clinician prior to CST. Implications for practice and training are discussed. Communication has been recognized as a central element of cancer care [1]. Ineffective communication may contribute to patients' confusion, uncertainty, and increased difficulty in asking questions, expressing feelings, and understanding information [2, 3], and may also contribute to clinicians' lack of job satisfaction and emotional burnout [4]. Therefore, communication skills trainings (CST) for oncology clinicians have been widely developed over the last decade.
\end{abstract}

\footnotetext{
M. Bernard $\cdot$ Y. de Roten $(\bowtie) \cdot$ J.-N. Despland Institut Universitaire de Psychothérapie, Centre Hospitalier Universitaire Vaudois and University of Lausanne,

Avenue de Morges 10,

CH-1004 Lausanne, Switzerland

e-mail: Yves.deRoten@chuv.ch

F. Stiefel

Service of Liaison Psychiatry, Centre Hospitalier Universitaire Vaudois and University of Lausanne,

Lausanne, Switzerland
}

These trainings should increase the skills of clinicians to respond to the patient's needs, and enhance an adequate encounter with the patient with efficient exchange of information [5]. While CSTs show a great diversity with regard to their pedagogic approaches [6,7], the main elements of CST consist of (1) role play between participants, (2) analysis of videotaped interviews with simulated patients, and (3) interactive case discussion provided by participants. As recently stated in a consensus paper [8], CSTs need to be taught in small groups (up to 10-12 participants) and have a minimal duration of at least 3 days in order to be effective. Several systematic reviews evaluated the impact of CST on clinicians' communication skills [9-11]. Effectiveness of CST can be assessed by two main approaches: participantbased and patient-based outcomes. Measures can be selfreported, but, according to Gysels et al. [10], behavioral assessment of patient-physician interviews [12] is the most objective and reliable method for measuring change after training. Based on 22 studies on participants' outcomes, Merckaert et al. [9] reported an increase of communication skills and participants' satisfaction with training and changes in attitudes and beliefs. The evaluation of CST remains a challenging task and variables mediating skills improvement remain unidentified. We recently thus conducted a study evaluating the impact of CST on clinicians' defenses by comparing the evolution of defenses of clinicians participating in CST with defenses of a control group without training [13]. Defenses are unconscious psychological processes which protect from anxiety or distress. Therefore, they contribute to the individual's adaptation to stress [14]. Perry refers to the term "defensive functioning" to indicate the degree of adaptation linked to the use of a range of specific defenses by an individual, ranging from low defensive functioning when he or she tends to use generally less adaptive defenses (such as projection, denial, or acting out) 
to high defensive functioning when he or she tends to use generally more adaptive defenses (such as altruism, intellectualization, or introspection) $[15,16]$. Although several authors have addressed the emotional difficulties of oncology clinicians when facing patients and their need to preserve themselves [7, 17, 18], no research has yet been conducted on the defenses of clinicians. For example, repeated use of less adaptive defenses, such as denial, may allow the clinician to avoid or reduce distress, but it also diminishes his ability to respond to the patient's emotions, to identify and to respond adequately to his needs, and to foster the therapeutic alliance. Results of the above-mentioned study [13] showed two groups of clinicians: one with a higher defensive functioning and one with a lower defensive functioning prior to CST. After the training, a difference in defensive functioning between clinicians who participated in CST and clinicians of the control group was only showed for clinicians with a higher defensive functioning. Some clinicians may therefore be more responsive to CST than others. To further address this issue, the present study aimed to evaluate the relationship between the level of adherence to an "ideally conducted interview", as defined by the teachers of the CST, and the level of the clinician' defensive functioning. We hypothesized that, after CST, clinicians with a higher defensive functioning show a greater adherence to the "ideally conducted interview" than clinicians with a lower defensive functioning.

\section{Keywords Communication Skills Training · Defence . Competence $\cdot$ Clinician}

\section{Methods}

Sample

The sample consisted of 31 clinicians (13 oncology physicians and 18 nurses) randomly selected from a pool of participants of a CST which took place in the Frenchspeaking part of Switzerland. The training is part of a national CST, which is conducted over 2 days, followed by individual supervisions and a follow-up meeting 6 months later. The CST is provided in small groups of about ten participants. Details of the training are described elsewhere [19]. Twenty-four clinicians were female $(77.4 \%)$, the mean age was $37.5(\mathrm{SD}=7.8)$, and the mean number of years of clinical experience was $11.33(\mathrm{SD}=6.2)$.

\section{Procedure}

Each clinician conducted two videotaped interviews with simulated patient, one at the beginning of the CST $(N=31)$, and one 6 months later at the end of the training $(N=31)$.
The simulated patients were played by three actors. Short written instructions were given to the clinician prior to the interviews, specifying the type of situation (curative or palliative) and the objectives of the interview. For the physicians, the objectives were to announce the diagnosis of a curable cancer, lymphoma, or testicular cancer or to propose palliative chemotherapy for stomach cancer. For the nurses, the objectives were to prepare the patient for curative or palliative chemotherapy. We controlled that there was no statistically significant effect of the actors or the scenarios on the adherence rating. Therefore, results could not be explained by differences of either the personal characteristics of the simulated patients or the affective load elicited by the scenarios.

Instruments

\section{Adherence}

First, a so-called ideal prototype of patient-interview was created by using the Psychotherapy Process Q-set (PQS) [20], a 105-item instrument which allows a comprehensive description in clinically relevant terms of a patient-clinician interaction. The prototype was based on the rating of eight of the nine teachers of the Swiss National Task Force of CST in oncology.

Following the procedure defined by the authors of the PQS, these eight ratings were then analyzed using a Q-type factor analysis [20, 21]. This factor analysis was labeled Q-type, because, contrary to the more familiar R-factor analysis, the data matrix was transposed so that the eight CST experts represented variables to be correlated over the 105 items of the PQS $(N=105)$. The experts' ratings were then subjected to a principal components factor analysis. By using a Q-type factor analysis, the prototypes are defined according to the Qfactors or principal components that emerge from the correlations among experts. Factor scores represented the weighted sum of each Q-item of the PQS. This procedure is based on the same methodology which was applied to PQS expert ratings to develop prototypes of ideal psychotherapeutic processes for a range of theoretical perspectives [22].

Second, PQS ratings of each of the 62 interviews with simulated patients were conducted by two trained research assistants. A consensus rating of $20 \%$ of the ratings $(N=12)$ revealed that reliability was only moderate with a mean ICC $(2,1)=0.50(\mathrm{SD}=0.25)$.

The adherence score was calculated as the correlation between each session rating and the factorial scores of the ideal prototype (see $[15,16]$ for more details of the statistical procedure).

\section{Clinician's Defenses}

The defense mechanism rating scales for clinician (DMRSC) was developed by Despland et al. [23] to assess 
clinicians' defenses. Based on the defense mechanism rating scale [24], a reliable and validated instrument used in psychotherapy research $[25,26]$, the DMRS-C identifies 30 independent defense mechanisms assigned to seven hierarchical levels of defensive functioning, according to their level of adaptation. Examples of high adaptive defenses are sublimation, humor, or self-affirmation and of low adaptive defenses are acting out, denial, or projection.

Defensive functioning is weighted according to its level of adaptation and an overall defensive functioning (ODF) score can be computed varying from one (low defensive functioning) to seven (high defensive functioning). Ratings were conducted by four coders (three authors of the DMRS-C and one trained coder), after reliability assessment on $20 \%$ of the cases revealed a mean $\operatorname{ICC}(2,1)=0.76(\mathrm{SD}=0.19)[13]$.

\section{Data Analyses}

The comparison between clinicians with lower and higher defensive functioning was carried out by using a univariate analysis of variance by controlling the adherence to the CSTprototype before training. All analyses were computed using the statistical software SPSS 15.0 and $\mathrm{G}^{*}$ power 3.0. Cohen's $d$ effect sizes were computed for the ANOVA and the $t$ tests [27]. All tests were two-tailed and the alpha was set at 0.05 .

\section{Results}

The mean ODF before training was $M=4.4$ ( $\mathrm{SD}=0.6)$. Accordingly, the sample was divided into two sub-groups: (1) high defensive functioning clinicians with $\mathrm{ODF} \geq 4.4(N=$ 15 ) and (2) low defensive functioning clinicians with $\mathrm{ODF}<$ $4.4(N=16)$.

Table 1 shows that physicians and nurses were equally represented in high and low defensive functioning categories $($ Chi2 $(1)=0.883, \mathrm{~ns})$. There was also no difference in terms of gender $(\operatorname{chi} 2(1)=1.006, n s)$, age $(t(29)=0.040, n s)$, years of experience $(t(29)=1.031, \mathrm{~ns})$, and adherence score before $(t(29)=-1.903, \mathrm{~ns})$ and after the training $(t(29)=0.030, \mathrm{~ns})$.

By taking into account the total sample, Fig. 1 shows that before CST, the two groups showed the same adherence score $(t(29=-0.610, p>0.05, d=0.24)$. After CST, clinicians with a higher defensive functioning showed a better adherence score to the prototype than clinicians with a lower defensive functioning when controlling for the adherence before $\operatorname{CST}(F(1,29)=5.258, p=0.030, d=0.42)$.

\section{Discussion}

After CST, clinicians with a higher defensive functioning (use of more adaptive defenses) showed a better adherence to the prototype of an "ideally conducted interview" than clinicians with a lower defensive functioning (use of less adaptive defenses). This is in line with our previous study [13] and confirms that some clinicians are more responsive to CST than others. Maerckart [9] and Butow [28] observed a persistent difficulty in identifying emotional cues of patients by some of the CST-trained participants. According to our data, clinicians with less adaptive defenses, like projection or denial, may not adequately perceive the external reality (i.e., the subjective reality of the patient) favoring self-protecting strategies.

Since there was no difference between nurses and physicians in terms of defensive functioning and adherence as well as controlled variables (age, gender, experience), defensive functioning may be considered as a common underlying mechanism - self-protective psychological mechanisms triggered by the affective load associated with the encounter with the patient - which mediates improvement of communication skills after training.

Fallowfield and Jenkins [7] already reported that the use of role plays and videos in CST provokes a great deal of anxiety for some health professionals. Our results call for a reflection on the design of CST (e.g., increased focus on professional identity, affective load, and defense

Table 1 Adherence at pre- and post-training according to professions, gender, and clinician's defensive functioning

\begin{tabular}{|c|c|c|c|c|c|c|}
\hline & \multicolumn{6}{|c|}{ Clinician's defensive functioning } \\
\hline & \multicolumn{3}{|c|}{ Low } & \multicolumn{3}{|c|}{ High } \\
\hline & $N$ & Pre-training $M(\mathrm{SD})$ & Post-training $M(\mathrm{SD})$ & $N$ & Pre-training $M(\mathrm{SD})$ & Post-training M (SD) \\
\hline Physicians & 5 & $0.45(0.20)$ & $0.27(0.13)$ & 8 & $0.33(0.21)$ & $0.39(0.22)$ \\
\hline Nurses & 10 & $0.16(0.23)$ & $0.24(0.30)$ & 8 & $0.29(0.26)$ & $0.44(0.12)$ \\
\hline Men & 1 & $0.27(-)$ & $0.75(-)$ & 3 & $0.40(0.29)$ & $0.46(0.17)$ \\
\hline Women & 14 & $0.25(0.27)$ & $0.20(0.21)$ & 13 & $0.28(0.22)$ & $0.41(0.18)$ \\
\hline
\end{tabular}

$M$ mean adherence score (calculated as the mean correlation with the CST ideal prototype) 


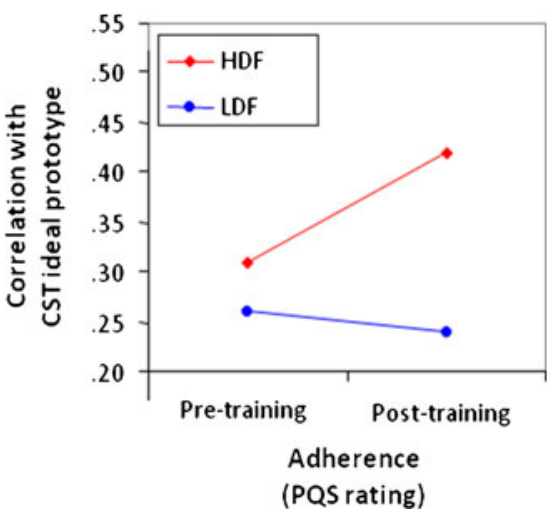

Fig. 1 Correlation with adherence at pre- and post-training for clinicians with high and low defensive functioning. HDL High defensive functioning, LDF Low defensive functioning

mechanisms of clinicians) and challenge their approach, which is rather independent of the participants' needs, limits, and resources. Based on our clinical experience, individual supervision is an effective tool to confront the clinicians of their defensive functioning and to reflect on more adequate ways to deal with emotionally loaded situations and different tasks in the patient encounter.

Several authors have already stressed the necessity to focus on the emotional dimension of patient interviews during CST [6-8]. To reach this goal, more research is needed, for example on the following issues: mediating mechanisms of clinician-patient communication with real patients; the impact of clinicians' defenses on clinical outcome (e.g., patient satisfaction, information recall, or therapeutic alliance), the relationship between clinicians' defenses and level of burnout, as well as psycho-physiological arousal.

Somme limitations of the present study have to be mentioned. The small sample size leads to an underpowered study, and results have to be considered as exploratory. The reliability of the PQS ratings (which is lower than expected [29]) is questionable and may have biased the results. Nevertheless, the methodology used to create an ideal prototype of CST proved to be useful in oncology because of its multi-dimensionality and its capacity to identify key dimensions of the patient-clinician interaction. Experts of CST in other countries will have to choose different items describing the ideal patient-clinician interview since communication is also influenced by cultural factors; uniformity in assessing communication outcome across nations is therefore neither realistic nor desirable.

In conclusion, this study showed that clinicians' defensive functioning is a mediating factor which may explain why clinicians do not equally benefit from CST. Confronting clinicians with their defensive functioning may help them to reflect on their personal way to relate to patients and to find more adequate ways to communicate in emotionally loaded patient encounters when breaking bad news.
Acknowledgments This study was supported by OncoSuisse, grant 01595-08-2004.

Conflict of Interest None

Ethical Consideration The ethics committee of the University Hospital of Lausanne considered it not necessary to evaluate this study since not patients, but healthy subjects, competent to decide on participation, were included in this study. Nevertheless, a hand-out with a description of the study was provided and a written informed consent was obtained from each participant. Privacy, confidentiality, and anonymity were guaranteed by first transcribing the videotaped interviews and coding them before the analysis. The video material will be destroyed at the end of the project and only the anonymous transcripts will be kept for 10 years.

\section{References}

1. Stiefel F (ed) (2006) Communication in Cancer Care. Springer Verlag, Berlin

2. Lerman C, Daly M, Walsh WP, Resch N, Seay J, Barsevick A, Birenbaum L, Heggan T, Martin G (1993) Communication between patients with breast cancer and health care providers: determinants and implications. Cancer 72:2612-620

3. Lamont EB, Christakis NA (2001) Prognostic disclosure to patients with cancer near the end of life. Ann Intern Med 134:1096-105

4. Sherman AC, Edwards D, Simonton S, Mehta P (2006) Caregiver stress and burnout in an oncology unit. Palliat Support Care 4:65-814

5. Butler L, Degner L, Baile W, Landry M (2005) Developing communication competency in the context of cancer: a critical interpretive analysis of provider training programs. Psychooncology 14:861-872

6. Maguire P, Pitceathly C (2002) Key communication skills and how to acquire them. BMJ 325:696-700

7. Fallowfield L, Jenkins V (2006) Current concepts of communication skills training in oncology. Recent Results Cancer Res 168:105-12

8. Stiefel F, Barth J, Bensing J, Fallowfield L, Jost L, Razzavi D (2010) Communication skills training in oncology: a position paper based on a consensus meeting among European experts in 2009. Ann Oncol. doi:10.1093/annonc/mdp564

9. Merckaert I, Libert Y, Delvaux N, Marchal S, Boniver J, Etienne AM, Klatersky J, Reynaert C, Scalliet P, Slachmuylder JL, Razavi D (2005) Factors that influence physicians' detection of distress in patients with cancer: can a communication skills program improve physicians' detection? Cancer 104:411-21

10. Gysels M, Richardson A, Higginson IJ (2005) Communication training for health professionals who care for patients with cancer: a systematic review of training methods. Support Care Cancer 13:356-66

11. Butler L, Degner L, Baile W, Landry M (2005) Developing communication competency in the context of cancer: a critical interpretive analysis of provider training programs. Psychooncology 14:861-72

12. Roter DL, Larson S (2002) The Roter interaction analysis system (RIAS): utility and flexibility for analysis of medical interaction. Patient Educ Couns 46:242-251

13. Bernard M, de Roten Y, Despland J-N, Stiefel F (2010) Communication skills training and clinicians' defenses in oncology: an exploratory, controlled study. Psychooncology 19:209-215

14. Perry JC (2001) A pilot study of defenses in psychotherapy of personality disorders entering psychotherapy. J Nerv Ment Dis 189:651-60

15. Vaillant GE (1992) Ego mechanisms of defense. Washington, American Psychiatric 
16. Perry JC, Cooper S (1989) An empirical study of defense mechanisms: i clinical interview and life vignette ratings. Arch Gen Psychiatry 46:444-452

17. Baile WF, Beale EA (2001) Giving bad news to cancer patients: matching process and content. J Clin Oncology 19:2575-77

18. Meier DE, Back AL, Morrison RS (2001) The inner life of physicians and care of the seriously ill. JAMA 286:3007-3014

19. Stiefel F, Bernhard J, Bianchi G, Dietrich L, Hürny C, Kiss A, Wössmer B (2012) CST: The swiss model. In: Kissane D, Bultz B, Butow P, Finlay I (eds) Handbook of communication in cancer and palliative care. Oxford University Press, London, In press

20. Ablon JS, Jones EE (1998) How expert clinicians' prototypes of an ideal treatment correlate with outcome in psychodynamic and cognitive-behavior therapy. Psychother Res 8:71-83

21. Ablon JS, Jones EE (2002) Validity of controlled clinical trials of psychotherapy: findings from the NIMH treatment of depression collaborative research program. Am J Psychiatry 159:775-783

22. Ablon JS, Jones EE (2000) Therapeutic action: a guide to psychoanalytic therapy. Jason Aronson, Northvale
23. Despland JN, Bernard M, Favre N, De Roten Y, Drapeau M, Stiefel F (2009) An empirical study of clinician's defenses. Psychol Psychother 82:73-81

24. Perry JC (1990) Defense mechanism rating scale, 5th edn. Cambridge University Press, New-York

25. Drapeau M, De Roten Y, Perry JC, Despland JN (2003) A study of stability and change in defense mechanisms during a brief psychodynamic investigation. J Nerv Ment Dis 191:496502

26. Hersoug AJ, Sexton HC, Hoglend PA (2002) Contribution of defensive functioning to the quality of working alliance and psychotherapy outcome. Am J Psychother 56:539-54

27. Cohen J (1988) Statistical power analysis for the behavioral sciences, 2nd edn. Lawrence Earlbaum Associates, Hillsdale

28. Butow P, Cockburn J, Girgis A, Bowman D, Schofield P, D'Este C, Stojanovski E, Tattersall MH, CUES Team (2008) Increasing oncologists' skills in eliciting and responding to emotional cues: evaluation of a communication skills training program. Psychooncology 17:209-18 